\title{
Implications of the Higgs discovery for supersymmetry
}

\author{
Abdelhak Djouadi ${ }^{1, a}$ \\ ${ }^{1}$ Laboratoire de Physique Théorique, U. Paris-Sud and CNRS, F-91405 Orsay, France.
}

\begin{abstract}
In the context of the minimal supersymmetric extension of the Standard Model, I summarize the implications of the discovery of the Higgs boson at the LHC with a mass of approximately $125 \mathrm{GeV}$. The impact of the measured mass and production/decay rates of the observed particle and of the constraints in the search for the heavier Higgs states at the LHC are discussed.
\end{abstract}

\section{Introduction}

The ATLAS and CMS historical discovery of a particle with a mass of approximately $125 \mathrm{GeV}$ [1] and properties that are compatible [2,3] with those of a scalar Higgs boson [4, 5] has far reaching consequences not only for the Standard Model (SM) but also for new physics models beyond it. This is particularly true for supersymmetric theories (SUSY) [6] that are widely considered to be the most attractive SM extensions. In the minimal supersymmetric extension of the SM (MSSM), two Higgs doublet fields are required to break the electroweak symmetry, leading to a physical spectrum with five Higgs particles: two $\mathrm{CP}-$-even $h$ and $H$, a $\mathrm{CP}-$ odd $A$ and two charged $H^{ \pm}$states [5]. Two parameters are needed to describe the MSSM Higgs sector at tree-level: the pseudoscalar Higgs mass $M_{A}$ and the ratio of vacuum expectation values of the two Higgs fields $\tan \beta$ that is constrained to be in the range $1 \lesssim \tan \beta \lesssim 60$. For a heavy $A$ state, $M_{A} \gg M_{Z}$, one is in the decoupling regime in which the $h$ state is light and has couplings to fermions and gauge bosons that are $\mathrm{SM}$-like (the $\mathrm{CP}$-even mixing angle becomes $\alpha \approx \frac{\pi}{2}-\beta$ ) while the $H$ and $H^{ \pm}$bosons become mass degenerate with $A, M_{H} \approx M_{H^{ \pm}} \approx M_{A}$, and decouple from W/Z bosons. The MSSM Higgs sector then looks almost exactly as the SM one.

There is however a major difference: while the SM Higgs mass was a free parameter, the mass of the lightest $h$ state is bounded from above and, depending on the SUSY parameters that enter the important radiative corrections, is restricted to $M_{h}^{\max } \approx 90-130 \mathrm{GeV}$ [7] if one assumes a SUSYbreaking scale $M_{S} \lesssim O(1 \mathrm{TeV})$ in order to avoid too much fine-tuning in the model. The requirement that the MSSM $h$ boson coincides with the one observed at the LHC, i.e. with $M_{h} \approx 125 \mathrm{GeV}$ and almost SM-like couplings as the LHC data indicate [2, 3], places strong constraints on the SUSY parameters, in particular the scale $M_{S}$. This comes in addition to the limits that have been obtained from the negative searches at the LHC of the heavier Higgs states and the supersymmetric particles.

In this talk, I summarise the implications of the LHC results for the MSSM. I first discuss the consequences of the $M_{h}$ measured value for the various unconstrained and constrained versions of the MSSM, and the impact of the measured $h$ production and decay rates on the SUSY parameters. The impact of the negative search of the heavy $H, A$ and $H^{ \pm}$states is then summarized.

ae-mail: abdelhak.djouadi@th.u-psud.fr 


\section{Implications of the Higgs mass value}

\subsection{The Higgs masses in the MSSM}

In the MSSM, the tree-level CP-even $h$ and $H$ boson masses depend only on $M_{A}$ and $\tan \beta$. However, many MSSM parameters such as the masses of third generation stop and sbottom squarks $m_{\tilde{t}_{i}}, m_{\tilde{b}_{i}}$ and their trilinear couplings $A_{t}, A_{b}$ enter through radiative corrections. The mass matrix is given by

$$
\mathcal{M}^{2}=M_{Z}^{2}\left(\begin{array}{cc}
c_{\beta}^{2} & -s_{\beta} c_{\beta} \\
-s_{\beta} c_{\beta} & s_{\beta}^{2}
\end{array}\right)+M_{A}^{2}\left(\begin{array}{cc}
s_{\beta}^{2} & -s_{\beta} c_{\beta} \\
-s_{\beta} c_{\beta} & c_{\beta}^{2}
\end{array}\right)+\left(\begin{array}{cc}
\Delta \mathcal{M}_{11}^{2} & \Delta \mathcal{M}_{12}^{2} \\
\Delta \mathcal{M}_{12}^{2} & \Delta \mathcal{M}_{22}^{2}
\end{array}\right)
$$

where we use the short-hand notation $s_{\beta} \equiv \sin \beta$ etc... and introduce the radiative corrections by a general $2 \times 2$ matrix $\Delta \mathcal{M}_{i j}^{2}$. One can then easily derive the neutral $\mathrm{CP}$ even Higgs boson masses and the mixing angle $\alpha$ that diagonalises the $h$ and $H$ states. The by far leading one-loop corrections to the mass matrix are controlled by the top Yukawa coupling $\lambda_{t}=m_{t} / v \sin \beta$ with $v=246 \mathrm{GeV}$, which appears with the fourth power; for a detailed discussion of these corrections, see for instance Ref. [7]. One obtains a very simple expression for $\Delta \mathcal{M}_{i j}^{2}$ if only this contribution is taken into account

$$
\Delta \mathcal{M}_{11}^{2} \sim \Delta \mathcal{M}_{12}^{2} \sim 0, \Delta \mathcal{M}_{22}^{2} \sim \frac{3 \bar{m}_{t}^{4}}{2 \pi^{2} v^{2} \sin ^{2} \beta}\left[\log \frac{M_{S}^{2}}{\bar{m}_{t}^{2}}+\frac{X_{t}^{2}}{M_{S}^{2}}\left(1-\frac{X_{t}^{2}}{12 M_{S}^{2}}\right)\right]
$$

where $M_{S}$ is the geometric average of the two stop masses $M_{S}=\sqrt{m_{\tilde{t}_{1}} m_{\tilde{t}_{2}}}$ defined to be the SUSYbreaking scale and $X_{t}$ the stop mixing parameter given by $X_{t}=A_{t}-\mu / \tan \beta$ with $\mu$ the higgsino mass parameter; $\bar{m}_{t}$ is the running $\overline{\mathrm{MS}}$ top mass to account for the leading two-loop QCD corrections. Other soft SUSY-breaking parameters, in particular $\mu$ and $A_{b}$ (and in general the corrections controlled by the bottom-Yukawa coupling $\lambda_{b}=m_{b} / v \cos \beta$ which at large $\mu \tan \beta$ become relevant) as well as the gaugino mass parameters $M_{1,2,3}$, provide a small correction to $\Delta \mathcal{M}_{i j}^{2}$.

The maximal value of the $h$ mass, $M_{h}^{\max }$ is given in the leading one-loop approximation above by $M_{h}^{2} \rightarrow M_{Z}^{2} \cos ^{2} 2 \beta+\Delta \mathcal{M}_{22}^{2}$ and is obtained for the choice of parameters [7]: i) a decoupling regime with heavy $A$ states, $M_{A} \sim O(\mathrm{TeV})$; ii) large $\tan \beta$ values, $\tan \beta \gtrsim 10$; iii) heavy stops, i.e. large $M_{S}$ values and we choose in general $M_{S} \leq 3 \mathrm{TeV}$ to avoid a too large fine-tuning; iv) a stop mixing parameter $X_{t}=\sqrt{6} M_{S}$, the so-called maximal mixing scenario that maximizes the stop loops [8]. If the parameters are optimized as above, the maximal $M_{h}$ value can reach the level of $M_{h}^{\max } \approx 130 \mathrm{GeV}$.

It was pointed out in Refs. [9-11] that when the measured value $M_{h}=125 \mathrm{GeV}$ is taken into account, the MSSM Higgs sector with solely the dominant radiative corrections included, can be again described with only the two basic parameters $\tan \beta$ and $M_{A}$ as it was the case at tree-level. More specifically, assuming that in the radiatively corrected $2 \times 2 \mathrm{CP}$-even Higgs mass matrix, the $\Delta \mathcal{M}_{22}^{2}$ entry is dominant, $\Delta \mathcal{M}_{22}^{2} \gg \Delta \mathcal{M}_{11}^{2}, \Delta \mathcal{M}_{12}^{2}$ (in Ref. [11], it has been shown that this is indeed the case in a large area of the MSSM space), one can simply trade the radiative corrections that involve the dependence on the numerous MSSM parameters, for the measured value $M_{h}=125 \mathrm{GeV}$ and obtain

$$
M_{H}^{2}=\frac{\left(M_{A}^{2}+M_{Z}^{2}-M_{h}^{2}\right)\left(M_{Z}^{2} c_{\beta}^{2}+M_{A}^{2} s_{\beta}^{2}\right)-M_{A}^{2} M_{Z}^{2} c_{2 \beta}^{2}}{M_{Z}^{2} c_{\beta}^{2}+M_{A}^{2} s_{\beta}^{2}-M_{h}^{2}}, \alpha=-\arctan \left(\frac{\left(M_{Z}^{2}+M_{A}^{2}\right) c_{\beta} s_{\beta}}{M_{Z}^{2} c_{\beta}^{2}+M_{A}^{2} s_{\beta}^{2}-M_{h}^{2}}\right)
$$

while for the mass of the $H^{ \pm}$boson, one can simply write $M_{H^{ \pm}}^{2}=M_{A}^{2}+M_{W}^{2}$ to a good approximation. This scenario, called hMSSM in Ref. [11], allows thus to ignore the radiative corrections to the Higgs masses and their complicated dependence on the MSSM parameters and to use simple formulae to derive the other parameters of the Higgs sector, $\alpha, M_{H}$ as well as $M_{H^{ \pm}}$. This considerably simplifies phenomenological analyses in the MSSM Higgs sector which up to now rely either on large scans of the parameter space (as will be seen in the next subsections) or resort to benchmark scenarios in which most of the MSSM parameters are fixed (as is the case of e.g. Ref. [8]). 


\subsection{Implications in the phenomenological MSSM}

In an unconstrained MSSM, analyses can be performed in the so-called "phenomenological MSSM" (pMSSM) [12], in which CP conservation, flavour diagonal sfermion mass and coupling matrices and universality of the first and second generations are imposed. The pMSSM involves 22 free parameters in addition to those of the SM: besides $\tan \beta$ and $M_{A}$, these are the higgsino mass $\mu$, the three gaugino masses $M_{1,2,3}$, the diagonal left- and right-handed sfermion mass parameters $m_{\tilde{f}_{L, R}}$ and the trilinear sfermion couplings $A_{f}$. Fortunately and as previously seen, besides $\tan \beta$ and $M_{A}$, only two of them play a major role: $M_{S}$ and $X_{t}$. The value of $M_{h}^{\max }$ can be maximized by tuning the MSSM parameters as discussed previously but, to be more precise, one needs to include all possible corrections.

In Ref. [13], an estimate of the upper bound on $M_{h}$ has been obtained using the program Suspect [12] which calculates the Higgs and superparticle spectrum in the MSSM including the most up-todate information and corrections. A large scan of the PMSSM 22 parameter space has been performed in an uncorrelated way in the following wide domains:

$$
\begin{array}{r}
1 \leq \tan \beta \leq 60,50 \mathrm{GeV} \leq M_{A} \leq 3 \mathrm{TeV},-9 \mathrm{TeV} \leq A_{f} \leq 9 \mathrm{TeV}, \\
50 \mathrm{GeV} \leq m_{\tilde{f}_{L}}, m_{\tilde{f}_{R}}, M_{3} \leq 3 \mathrm{TeV}, 50 \mathrm{GeV} \leq M_{1}, M_{2},|\mu| \leq 1.5 \mathrm{TeV} .
\end{array}
$$

The results are shown in Fig. 1 where, in the left-hand side, the obtained maximal value $M_{h}^{\max }$ is displayed as a function of the ratio of parameters $X_{t} / M_{S}$. The resulting values are confronted to the mass range $123 \mathrm{GeV} \leq M_{h} \leq 127 \mathrm{GeV}$ when the parametric uncertainties from the SM inputs and the theoretical uncertainties in the determination of $M_{h}$, that are assumed to be of $2 \mathrm{GeV}$, are included.

For $M_{S} \approx 1 \mathrm{TeV}$, only the scenarios with large $X_{t} / M_{S}$ values and, in particular, those close to the maximal mixing scenario $X_{t} / M_{S} \approx \sqrt{6}$ survive. The no-mixing scenario $X_{t} \approx 0$ is ruled out for $M_{S} \leq 3 \mathrm{TeV}$, while the typical mixing scenario, $X_{t} \approx M_{S}$, needs large $M_{S}$ and moderate to large $\tan \beta$. We obtain $M_{h}^{\max }=136,123$ and $126 \mathrm{GeV}$ with maximal, zero and typical mixing.
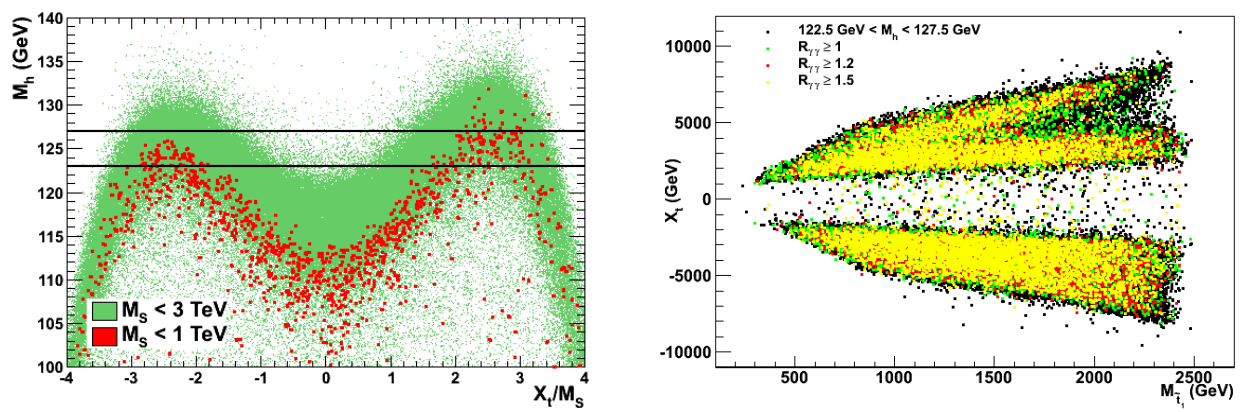

Figure 1. The maximal value of the $h$ boson mass as a function of $X_{t} / M_{S}$ in the pMSSM when all other soft SUSY-breaking parameters and $\tan \beta$ are scanned (left) and the contours for $123<M_{h}<127 \mathrm{GeV}$ in the $\left[m_{\tilde{t}_{1}}, X_{t}\right]$ plane for some selected range of $\tan \beta$ values (right). From Ref. [13].

The right-hand side of Fig. 1 shows the contours in the $\left[m_{\tilde{t}_{1}}, X_{t}\right]$ plane where we obtain the mass range $123 \mathrm{GeV}<M_{h}<127 \mathrm{GeV}$ from our pMSSM scan with $X_{t} / M_{S} \leq 3$; the regions in which $\tan \beta \leq 3,5$ and 60 are highlighted. One sees again that a large part of the parameter space is excluded if the Higgs mass constraint is imposed. In particular, large $m_{\tilde{t}_{1}}$ values, in general corresponding to large $M_{S}$ are favored. However, the possibility that $m_{\tilde{t}_{1}}$ is of the order of a few hundred $\mathrm{GeV}$ is still allowed, provided that stop mixing (which leads to a significant $m_{\tilde{t}_{1}}, m_{\tilde{t}_{2}}$ splitting) is large. Thus light stops (and electroweak sparticles) can be still searched for at the LHC. Note that generic squark as well as gluino masses above $1 \mathrm{TeV}$ are also required by the direct searches of SUSY particles at the LHC, confirming the need of high $M_{S}$ values to cope with $M_{h} \approx 125 \mathrm{GeV}$. 


\subsection{Implications for constrained MSSM scenarios}

In constrained MSSM scenarios (cMSSM), the various soft SUSY-breaking parameters obey a number of universal boundary conditions at a high energy scale, thus reducing the number of basic input parameters to a handful. These inputs are evolved via the MSSM renormalisation group equations down to the low energy scale $M_{S}$ where the conditions of proper electroweak symmetry breaking (EWSB) are imposed. Three classes of such models have been widely discussed in the literature: i) the minimal supergravity (mSUGRA) model in which SUSY-breaking is assumed to occur in a hidden sector which communicates with the visible sector only via flavour-blind gravitational interactions, leading to universal soft breaking terms ie a common $m_{1 / 2}, m_{0}, A_{0}$ values for the gaugino, sfermion masses and trilinear couplings, $i$ ) the gauge mediated SUSY-breaking (GMSB) model in which SUSY-breaking is communicated to the visible sector via gauge interactions, iii) the anomaly mediated SUSY-breaking (AMSB) model in which SUSY-breaking is communicated to the visible sector via a super-Weyl anomaly. For a review of these scenarios see for instance Ref. [6].

These models are described by $\tan \beta$, the sign of $\mu$ and a few continuous parameters. We adopt the following ranges for the input parameters of these scenarios:

$\begin{array}{rccc}\text { mSUGRA: } & 50 \mathrm{GeV} \leq m_{0} \leq 3 \mathrm{TeV}, & 50 \mathrm{GeV} \leq m_{1 / 2} \leq 3 \mathrm{TeV}, & \left|A_{0}\right| \leq 9 \mathrm{TeV} \\ \text { GMSB: } & 10 \mathrm{TeV} \leq \Lambda \leq 1000 \mathrm{TeV}, & 1 \leq M_{\text {mess }} / \Lambda \leq 10^{11}, & N_{\text {mess }}=1 \\ \text { AMSB: } & 1 \mathrm{TeV} \leq m_{3 / 2} \leq 100 \mathrm{TeV}, & 50 \mathrm{GeV} \leq m_{0} \leq 3 \mathrm{TeV} & \end{array}$

allow for both signs of $\mu$, require $1 \leq \tan \beta \leq 60$ and, to avoid the need for excessive fine-tuning in the EWSB conditions, impose an additional bound $M_{S}=M_{\mathrm{EWSB}}=\sqrt{m_{\tilde{t}_{1}} m_{\tilde{t}_{2}}}<3 \mathrm{TeV}$. In the case of mSUGRA, one can study special cases such as: no-scale scenario with $m_{0} \approx A_{0} \approx 0, m_{0} \approx 0$ and $A_{0} \approx-\frac{1}{4} m_{1 / 2}$ which approximately corresponds to the constrained next-to-MSSM (cNMSSM), $A_{0} \approx-m_{0}$ which corresponds to a very constrained MSSM (VCMSSM), and a non-universal Higgs mass model (NUHM) in which the universal soft SUSY-breaking scalar mass terms are different for the sfermions and for the two Higgs doublet fields. Details are given in Ref. [13].

Using again the program Suspect, we have performed a full scan of these scenarios. The results for $M_{h}^{\max }$ are shown in the left-hand side of Fig. 2 as a function of $\tan \beta$, the input parameter that is common to all models, and in the right-hand side of the figure as a function of $M_{S}$.
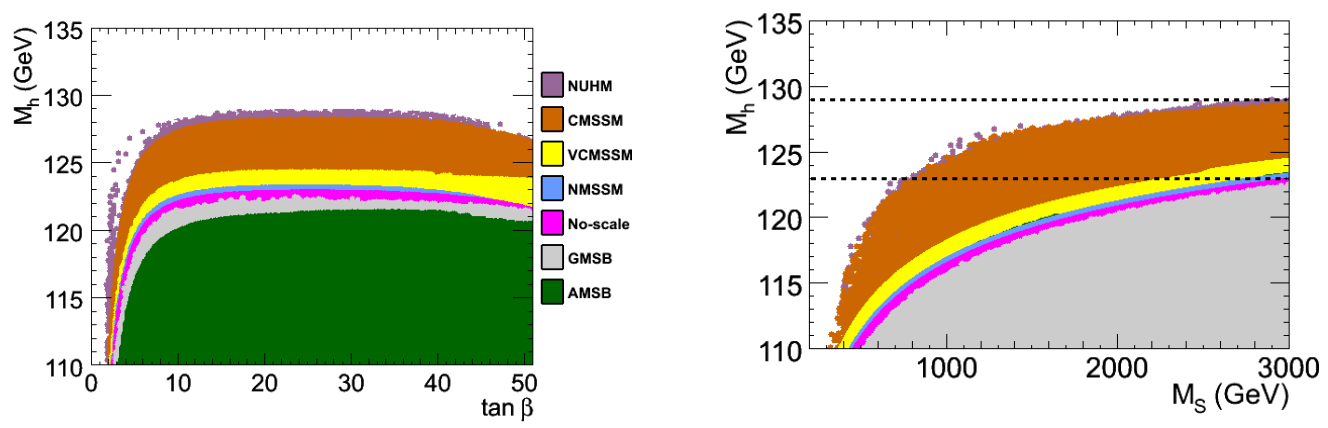

Figure 2. $M_{h}^{\max }$ as a function of $\tan \beta$ (left) and $M_{S}$ (right) with a scan of all other parameters in various constrained MSSM scenarios; the range $123<M_{h}<127 \mathrm{GeV}$ for the mass is highlighted. From Ref. [13].

In contrast to the pMSSM, the various parameters which enter the radiative corrections to the MSSM Higgs sector are not all independent in constrained scenarios as a consequence of the relations between SUSY breaking parameters that are set at the high-energy scale and the requirement that electroweak symmetry breaking is triggered radiatively for each set of input parameters which leads to additional constraints. Hence, it is not possible to freely tune the relevant weak-scale parameters to obtain the maximal value of $M_{h}$ given previously. In order to obtain a reliable determination of $M_{h}^{\max }$ 
in a given constrained SUSY scenario, it is necessary to scan through the allowed range of values for all relevant SUSY parameters. In all cases, the maximal $M_{h}$ value is obtained for $\tan \beta$ around 20 . We observe that in the adopted parameter space of the models and with the central values of the SM inputs, the upper $h$ mass value (rounded to the upper half $\mathrm{GeV}$ ) is $M_{h}^{\max }=121 \mathrm{GeV}$ in AMSB, i.e. much less that $125 \mathrm{GeV}$, while in the GMSB scenario one has $M_{h}^{\max }=121.5 \mathrm{GeV}$. Thus, clearly, the two scenarios are disfavoured if the lightest CP-even Higgs particle has indeed a mass in the range $123<M_{h}<127 \mathrm{GeV}$. In the case of mSUGRA, we obtain a maximal value $M_{h}^{\max }=128 \mathrm{GeV}$ and, thus, some parameter space of the model would still survive the $M_{h}$ constraint.

The upper bound on $M_{h}$ in each of these scenarios can be qualitatively understood by considering the allowed values of the trilinear coupling $A_{t}$ that determines the stop mixing parameter $X_{t}$ and thus $M_{h}$ for a given scale $M_{S}$. In GMSB, one has $A_{t} \approx 0$ at relatively low scales and its magnitude does not significantly increase in the evolution down to the scale $M_{S}$; this implies that we are almost in the no-mixing scenario which gives a low value of $M_{h}$ as can be seen from Fig. 1. In AMSB, one has a non-zero $A_{t}$ that is fully predicted at any scale in terms of the Yukawa and gauge couplings; however, the ratio $A_{t} / M_{S}$ with $M_{S}$ determined from the overall SUSY breaking scale $m_{3 / 2}$ turns out to be rather small, implying again that we are close to the no-mixing scenario. Finally, in the mSUGRA model, since we have allowed $A_{t}$ to vary in a wide range as $\left|A_{0}\right| \leq 9 \mathrm{TeV}$, one can get a large $A_{t} / M_{S}$ ratio which leads to a heavier Higgs particle. However, one cannot easily make $X_{t} / M_{S} \approx \sqrt{6}$ so that we are not in the maximal-mixing scenario and the higher pMSSM $M_{h}^{\max }$ in value is not reached.

\subsection{Split and high-scale SUSY models}

The previous discussion assumed a not too high SUSY-breaking scale, $M_{S} \lesssim 3 \mathrm{TeV}$, to avoid excessive fine-tuning. However, many scenarios with a high SUSY scale have been considered, a most popular one being the split-SUSY scenario. Here, one has a very large value of $M_{S}$ which implies that no sfermion is accessible at the LHC while the mass parameters for the gauginos and higgsinos can be kept at the weak scale, allowing for a solution to the dark matter problem and a successful gauge coupling unification. The SUSY solutions to these two remaining problems are abandoned if one takes the very extreme attitude of assuming that the gauginos and higgsinos have also a mass of the order of the SUSY scale, as is the case of the high-scale SUSY models.

In these SUSY scenarios, the $h$ particle is still light particle as, at the high scale $M_{S}$, the boundary condition on the quartic Higgs coupling of the theory is determined by SUSY:

$$
\lambda\left(M_{S}\right)=\left[g^{2}\left(M_{S}\right)+g^{\prime 2}\left(M_{S}\right)\right] \cos ^{2} 2 \beta / 4
$$

where $g$ and $g^{\prime}$ are the SU(2) and U(1) gauge couplings. One can thus have an $h$ boson with a mass close to $125 \mathrm{GeV}$ for a given choice of the parameters $M_{S}$ and $\tan \beta$. Nevertheless, if the scalars are very heavy, they will lead to radiative corrections that are enhanced by $\operatorname{large} \log \left(M_{S} / M_{Z}\right)$ terms and, in order to have reliable predictions, one has to properly decouple the heavy states from the low-energy theory and resum the large logarithmic corrections. In addition, the radiative corrections due to the gauginos and the higgsinos have to be implemented in the split-SUSY case. Following an early work done in Ref. [14] where a comprehensive study of the split SUSY spectrum has been performed and all the features of the model have been implemented in the code SuSpect [12], an analysis of these scenarios has been presented in Ref. [13] and is summarised in Fig. 3.

The figure shows that the requirement that the Higgs mass is in the range $123<M_{h}<127 \mathrm{GeV}$ imposes strong constraints on the parameters of these two models. For this Higgs mass range, very large scales are needed for $\tan \beta \approx 1$ in the split-SUSY and high-scale SUSY scenarios, while scales not too far from $M_{S} \approx 10^{4} \mathrm{GeV}$ are required at high $\tan \beta$. Thus, even in these extreme scenarios, SUSY could manifest itself at scales much below $M_{\mathrm{GUT}}$ if $M_{h} \approx 125 \mathrm{GeV}$. 

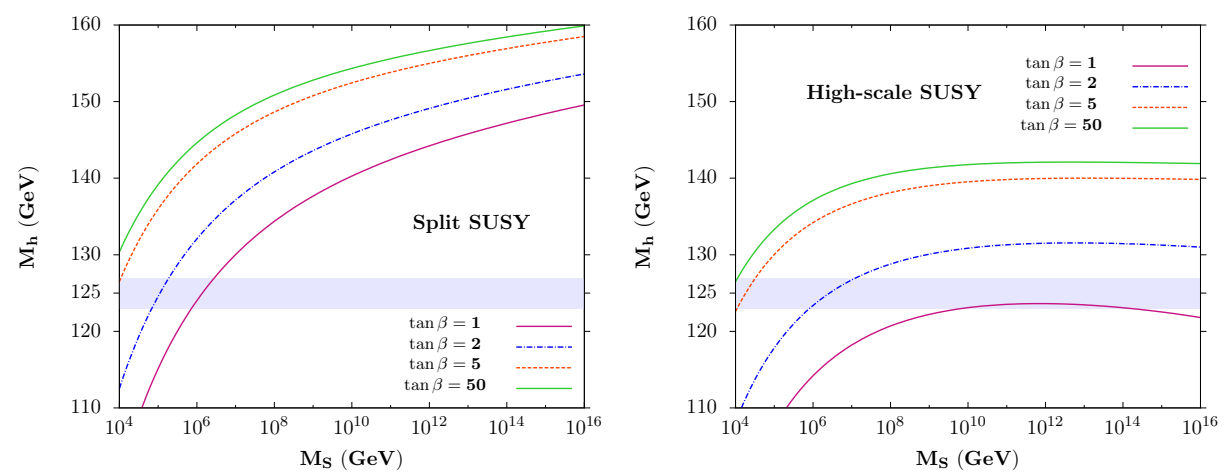

Figure 3. The value of $M_{h}$ as a function of $M_{S}$ for several values of $\tan \beta=1,2,5,50$ in the split SUSY (left) and high-scale SUSY (right) scenarios. From Ref. [13] and based on an analysis performed in Ref. [14].

\subsection{Splitting the Higgs and sfermion sectors}

In the previous high scale scenarios, the Higgs mass parameters were assumed to be related to those of the scalar fermions such that the heavier Higgs boson masses are of the same order as the high scale, $M_{A} \approx M_{S}$. However, this needs not to be true in general and one can decouple the Higgs masses from those of the sfermions. If one is primarily concerned with the MSSM Higgs sector, one can be conservative and allow any value for $M_{A}$ irrespective of $M_{S}$. This is the quite "model-independent" approach that has been advocated in Refs. [9, 11]: take $M_{A}$ as a free parameter with values ranging from $O(100 \mathrm{GeV})$ up to $O\left(M_{S}\right)$, but make no restriction on $M_{S}$ which can be set to any value.

An important consequence is the reopening of the low $\tan \beta$ region, $\tan \beta \lesssim 3$, that was long thought to be forbidden if one requires a SUSY scale $M_{S} \lesssim 1 \mathrm{TeV}$, as a result of the limit $M_{h} \gtrsim 114 \mathrm{GeV}$ from SM-like Higgs searches at LEP [2]. If $M_{S}$ is large enough, this small $\tan \beta$ region becomes viable again. To estimate the required magnitude of $M_{S}$, one can still use Suspect in which the possibility $M_{S} \gg 1 \mathrm{TeV}$ is implemented [14] with the full set of radiative corrections up to two-loops included. In Fig. 4, displayed are the contours in the $\left[\tan \beta, M_{S}\right]$ plane for fixed values $M_{h}=120-132 \mathrm{GeV}$ of the observed Higgs state (including uncertainties); $X_{t}=\sqrt{6} M_{S}$ is assumed.

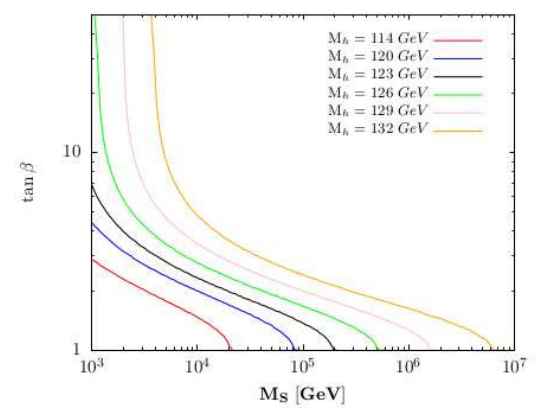

Figure 4. Contours for fixed values $M_{h}=120,123,126,129$ and $132 \mathrm{GeV}$ in the $\left[\tan \beta, M_{S}\right]$ plane in the decoupling limit $M_{A} \gg M_{Z}$; the "LEP2 contour" for $M_{h}=114 \mathrm{GeV}$ is also shown.

One observes that values of $\tan \beta \approx 1$ are possible and allow for an acceptable $M_{h}$ value provided the scale $M_{S}$ is large enough. For instance, while one can accommodate a scale $M_{S} \approx 1 \mathrm{TeV}$ with $\tan \beta \approx 5$, a large scale $M_{S} \approx 20 \mathrm{TeV}$ is required to obtain $\tan \beta \approx 2$; to reach the limit $\tan \beta=1$, an order of magnitude increase of $M_{S}$ will be required. Outside the decoupling regime, the obtained $M_{S}$ for a given $M_{h}$ value will be of course larger. For completeness, also shown is the contour for the LEP2 limit $M_{h}=114 \mathrm{GeV}$ which shows that $\tan \beta \approx 1$ is still allowed if $M_{S} \gtrsim 20 \mathrm{TeV}$. 


\section{Implications from the Higgs production and decay rates}

In the MSSM, the couplings of the CP-even particles $h$ and $H$ to gauge bosons and fermions, compared to the SM Higgs couplings, are changed by factors that involve the mixing angles $\beta$ and $\alpha$. Outside the decoupling regime where they reach unity, the reduced couplings (i.e. normalized to their $\mathrm{SM}$ values) of the lighter $h$ state to third generation $t, b, \tau$ fermions and gauge bosons $V=W / Z$ are

$$
c_{V}^{0}=\sin (\beta-\alpha), \quad c_{t}^{0}=\cos \alpha / \sin \beta, \quad c_{b}^{0}=-\sin \alpha / \cos \beta
$$

They thus depend not only on the two inputs $\left[\tan \beta, M_{A}\right]$ as it occurs at tree-level but, a priori, on the entire MSSM spectrum as a result of the radiative corrections, in the same way as the Higgs masses. In principle, as discussed earlier, knowing $\tan \beta$ and $M_{A}$ and fixing $M_{h}$ to its measured value, the couplings can be determined in general. However, this is true when only the radiative corrections to the Higgs masses are included. Outside the regime in which the pseudoscalar $A$ boson and the supersymmetric particles are very heavy, there are also direct radiative corrections to the Higgs couplings not contained in the mass matrix of eq. (1) and which can alter this simple picture. The impact of these corrections has been discussed in Refs. $[11,15]$ and is briefly summarized below.

First, in the case of $b$-quarks, additional vertex corrections modify the tree-level $h b \bar{b}$ coupling: they grow as $m_{b} \mu \tan \beta$ and can be very large at high $\tan \beta$. The dominant component comes from the SUSY-QCD corrections with sbottom-gluino loops that can be approximated by $\Delta_{b} \simeq 2 \alpha_{s} /(3 \pi) \times$ $\mu m_{\tilde{g}} \tan \beta / \max \left(m_{\tilde{g}}^{2}, m_{\tilde{b}_{1}}^{2}, m_{\tilde{b}_{2}}^{2}\right)$ [16]. Outside the decoupling regime the $c_{b}$ coupling reads

$$
c_{b} \approx c_{b}^{0} \times\left[1-\Delta_{b} /\left(1+\Delta_{b}\right) \times(1+\cot \alpha \cot \beta)\right]
$$

with $\tan \alpha \rightarrow-1 / \tan \beta$ for $M_{A} \gg M_{Z}$. A large $\Delta_{b}$ would significantly alter the dominant $h \rightarrow b \bar{b}$ partial width and affect the branching fractions of all other decay modes.

In addition, the $h t \bar{t}$ coupling is derived indirectly from the $g g \rightarrow h$ production cross section and the $h \rightarrow \gamma \gamma$ decay branching ratio, two processes that are generated by triangular loops. In the MSSM, these loops involve not only the top quark (and the $W$ boson in the decay $h \rightarrow \gamma \gamma$ ) but also contributions from supersymmetric particles, if not too heavy. In the case of $g g \rightarrow h$ production, only the contributions of stops is generally important. Including the later and working in the limit $M_{h} \ll m_{t}, m_{\tilde{t}_{1,2}}$, the coupling $c_{t}$ from the gg fusion process is approximated by [17]

$$
c_{t} \approx c_{t}^{0}\left[1+m_{t}^{2}\left(m_{\tilde{t}_{1}}^{2}+m_{\tilde{t}_{2}}^{2}-X_{t}^{2}\right) /\left(4 m_{\tilde{t}_{1}}^{2} m_{\tilde{t}_{2}}^{2}\right)\right]
$$

which shows that indeed, $\tilde{t}$ contributions can be very large for light stops and for large stop mixing. In the $h \rightarrow \gamma \gamma$ decay rate, because the $t, \tilde{t}$ electric charges are the same, the $h t \bar{t}$ coupling is shifted by the same amount. If one ignores the usually small contributions of the other sparticles, the $h t \bar{t}$ vertex can be simply parametrised by the effective coupling of eq. (8). We note that the $h$ couplings to $\tau$ leptons and $c$ quarks do not receive the direct corrections of eqs. (7) and (8) and one should still have $c_{c}=c_{t}^{0}$ and $c_{\tau}=c_{b}^{0}$. However, using $c_{t, b}$ or $c_{t, b}^{0}$ in this case has almost no impact in practice as these couplings appear only in the branching ratios for the decays $h \rightarrow c \bar{c}$ and $\tau^{+} \tau^{-}$which are small and the direct corrections should not be too large. One can thus, in a first approximation, assume that $c_{c}=c_{t}$ and $c_{\tau}=c_{b}$. Another caveat is due to the invisible Higgs decays which are assumed to be absent [18].

Hence, because of the direct corrections, the Higgs couplings cannot be described only by $\beta$ and $\alpha$ as in eq. (6). To characterize the Higgs particle at the LHC, it was advocated that at least three independent $h$ couplings should be considered, namely $c_{t}, c_{b}$ and $c_{V}=c_{V}^{0}$ [11]. One can thus define the following effective Lagrangian,

$$
\mathcal{L}_{h}=c_{V} g_{h W W} h W_{\mu}^{+} W^{-\mu}+c_{V} g_{h Z Z} h Z_{\mu}^{0} Z^{0 \mu}-c_{t} y_{t} h \bar{t}_{L} t_{R}-c_{t} y_{c} h \bar{c}_{L} c_{R}-c_{b} y_{b} h \bar{b}_{L} b_{R}-c_{b} y_{\tau} h \bar{\tau}_{L} \tau_{R}+\text { h.c. }
$$



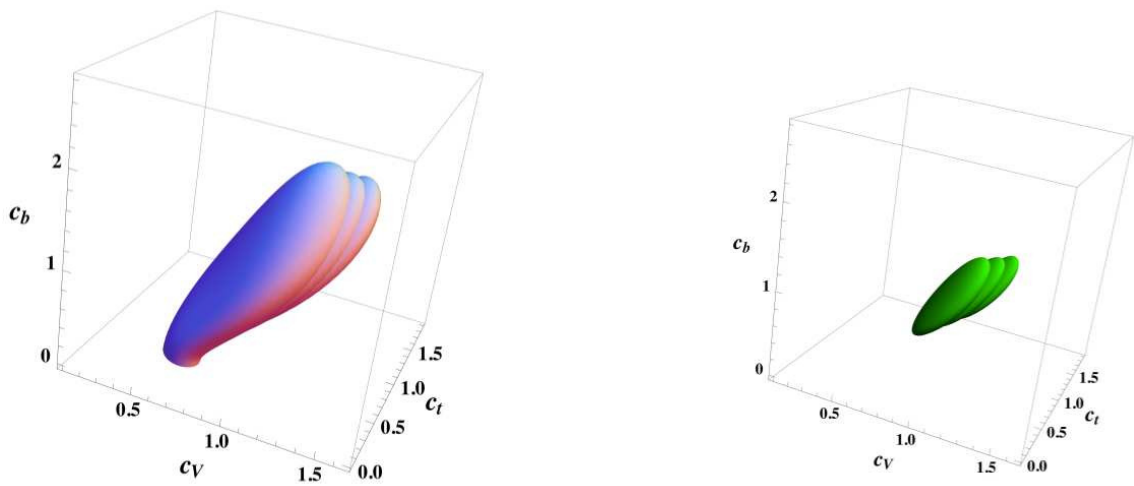

Figure 5. The best-fit region at 99\%CL (left) and 68\%CL (right) for the Higgs signal strengths in the $\left[c_{t}, c_{b}, c_{V}\right]$ space from Ref. [11]. The three overlapping regions are for the central and extreme choices of the theoretical prediction for the Higgs rates including uncertainties which are taken as a bias and not a nuisance.

where $y_{t, c, b, \tau}=m_{t, c, b, \tau} / v$ are the Yukawa couplings of the heavy SM fermions, $g_{h W W}=2 M_{W}^{2} / v$ and $g_{h Z Z}=M_{Z}^{2} / v$ the $h W W$ and $H Z Z$ couplings and $v$ the SM Higgs vev.

In Ref. [11], a three-dimensional fit of the $h$ couplings was performed in the space $\left[c_{t}, c_{b}, c_{V}\right]$, assuming $c_{c}=c_{t}$ and $c_{\tau}=c_{b}$ as discussed above and of course the custodial symmetry relation $c_{V}=$ $c_{W}=c_{Z}$ which holds in supersymmetric models. The results of this fit are presented in Fig. 5 for $c_{t}, c_{b}, c_{V} \geq 0$. The best-fit value for the couplings, with the $\sqrt{s}=7+8 \mathrm{TeV}$ ATLAS and CMS data turns out to be $c_{t}=0.89, c_{b}=1.01$ and $c_{V}=1.02$.
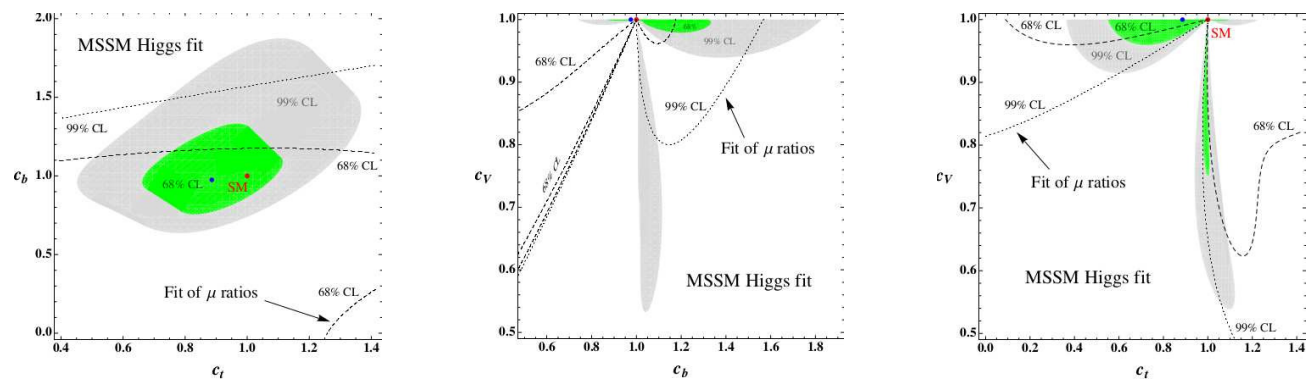

Figure 6. Best-fit regions at $68 \%$ and $99 \% \mathrm{CL}$ for the Higgs signal strengths and their ratios in the planes $\left[c_{t}, c_{b}\right]$, $\left[c_{t}, c_{V}\right]$ and $\left[c_{V}, c_{b}\right]$ with the best-fit point is indicated in blue; from Ref. [11].

In scenarios where the direct corrections in eqs. (7)-(8) are not quantitatively significant (i.e. considering either not too large values of $\mu \tan \beta$ or high sfermion masses), one can use the MSSM relations of eq. (6) to reduce the number of effective parameters down to only two. This allows to perform two-parameter fits in the planes $\left[c_{V}, c_{t}\right],\left[c_{V}, c_{b}\right]$ and $\left[c_{t}, c_{b}\right]$. As an example, the fit of the signal strengths and their ratios in the $\left[c_{t}, c_{b}\right]$ plane is displayed in Fig. 6. In this two-dimensional case, the best-fit point is located at $c_{t}=0.88$ and $c_{b}=0.97$, while $c_{V} \simeq 1$. Note that although for the best-fit point one has $c_{b} \lesssim 1$, actually $c_{b} \gtrsim 1$ in most of the $1 \sigma$ region.

Using the formulae eq. (3) for the angle $\alpha$ and using the input $M_{h} \approx 125 \mathrm{GeV}$, one can make a global fit in the plane $\left[\tan \beta, M_{A}\right]$. This is shown in Fig. 7 where the $68 \%, 95 \%$ and $99 \%$ CL contours from the signal strengths and their ratios are displayed when the theory uncertainty is taken as a bias. The best-fit point when the latter uncertainty is set to zero, is obtained for the values $\tan \beta=1$ and $M_{A}=557 \mathrm{GeV}$, which implies for the other parameters using $M_{h}=125 \mathrm{GeV}: M_{H}=580 \mathrm{GeV}$, $M_{H^{ \pm}}=563 \mathrm{GeV}$ and $\alpha=-0.837 \mathrm{rad}$ which leads to $\cos (\beta-\alpha) \simeq-0.05$. 


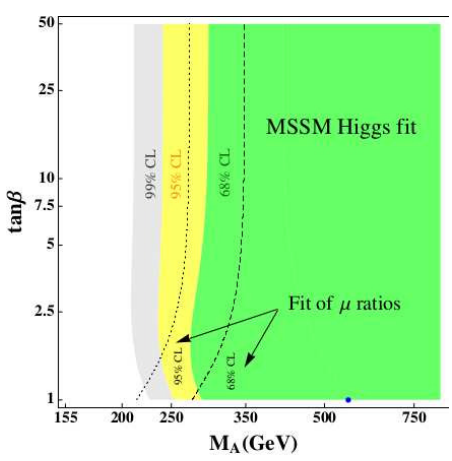

Figure 7. Best-fit regions (best point in blue) for the signal strengths and their ratios in the plane [ $\left.\tan \beta, M_{A}\right]$ [11].

Such a point with $\tan \beta \approx 1$ implies an extremely large value of the SUSY scale, $M_{S}=O(100)$ $\mathrm{TeV}$, for $M_{h} \approx 125 \mathrm{GeV}$. One should note, however, that the $\chi^{2}$ value is relatively stable all over the $1 \sigma$ region. Hence, larger values of $\tan \beta$ (and lower values of $M_{A}$ ) could also be accommodated reasonably well by the fit, allowing thus for not too large $M_{S}$ values. In all, cases one nevertheless has a limit $M_{A} \gtrsim 200 \mathrm{GeV}$ at the $99 \% \mathrm{CL}$ and even tighter for a lower level of confidence.

Note that questions like is there an impact of the SUSY particles in these couplings? is it possible that the heavier $H$ boson is instead the observed Higgs particle? is there is a discrepancy in the $h$ boson two-photon decay channel? or is there still some room for invisible decays into the lightest SUSY particles? have been asked with the answer being the same to all of them: not really.

\section{Implications from heavy Higgs searches}

We turn now to the constraints on the MSSM Higgs sector that can be obtained from the search of the heavier $H / A$ and $H^{ \pm}$states at the LHC and start with a summary of their production and decay rates.

\section{1 $\mathrm{H}, \mathrm{A}, \mathrm{H}^{ \pm}$decays and production at the $\mathrm{LHC}$}

The production and decay pattern of the MSSM Higgs bosons crucially depend on $\tan \beta$. In the high $\tan \beta$ regime, the couplings of the neutral $\Phi=H / A$ and $H^{ \pm}$bosons to $b$ quarks and $\tau$ leptons are so strongly enhanced, and the couplings to top quarks and massive gauge bosons suppressed, that the pattern is rather simple. First for the decays, the $\Phi \rightarrow t \bar{t}$ channel and all other modes are negligible and the $\Phi$ states decay almost exclusively into $\tau^{+} \tau^{-}$and $b \bar{b}$ pairs, with branching ratios of $\operatorname{BR}(\Phi \rightarrow \tau \tau) \approx 10 \%$ and $\operatorname{BR}(\Phi \rightarrow b \bar{b}) \approx 90 \%$. The $H^{ \pm}$boson decay into $\tau v_{\tau}$ final states with a branching fraction of almost $100 \%$ for $H^{ \pm}$masses below the $t b$ threshold, $M_{H^{ \pm}} \lesssim m_{t}+m_{b}$, and a branching ratio of only $\approx 10 \%$ above, while the rate of $H^{ \pm} \rightarrow t b$ is at the $\approx 90 \%$ level in most cases.

For the production, only two processes are relevant at high $\tan \beta: g g \rightarrow \Phi$ with the $b$-loop included and associated production with $b$-quarks, $g g / q \bar{q} \rightarrow b \bar{b}+\Phi$, which is equivalent to the fusion process $b \bar{b} \rightarrow \Phi$ with no-additional final $b$-quark. In both the $b \bar{b}$ and $g g$ fusion cases, as $M_{\Phi} \gg m_{b}$, chiral symmetry holds and the rates are approximately the same for the CP-even $H$ and odd $A$ states.

The most powerful search channel for the heavier MSSM Higgs particles at the LHC is by far the process $p p \rightarrow g g+b \bar{b} \rightarrow \Phi \rightarrow \tau^{+} \tau^{-}$. The precise values of the cross section times branching fraction for this process at the LHC have been updated in Refs. $[19,20]$ and an assessment of the associated theoretical uncertainties has been made. It turns out that, in the production cross section, the total uncertainty from scale variation, the PDFs and $\alpha_{s}$ as well as from the $b$-quark mass are not that small: $\Delta^{\mathrm{TH}} \sigma(p p \rightarrow \Phi) \times \mathrm{BR}(\Phi \rightarrow \tau \tau) \approx \pm 25 \%$ in the entire $M_{\Phi}$ range probed at the LHC at $\sqrt{s}=8 \mathrm{TeV}$. 
Besides the QCD uncertainty, the impact of the SUSY particle contributions on the $\sigma(p p \rightarrow \Phi \rightarrow \tau \tau)$ rate (either directly in the decays or indirectly in loops) has also been discussed and found to be small. One thus expects the rate to be robust and to depend almost exclusively on $M_{A}$ and $\tan \beta$.

For the charged Higgs boson, the dominant search channel is in $H^{ \pm} \rightarrow \tau v$ with the $H^{ \pm}$bosons produced in top quark decays for $M_{H^{ \pm}} \lesssim 160 \mathrm{GeV}, t \rightarrow H^{+} b \rightarrow \tau v b$. This is particularly true at high $\tan \beta$ values when $\operatorname{BR}\left(t \rightarrow H^{+} b\right)$ is significant. For higher $H^{ \pm}$masses, one should rely on the three-body production process $p p \rightarrow t b H^{ \pm} \rightarrow t b \tau v$ but the rates are presently rather small.

In the low $\tan \beta$ regime, $\tan \beta \lesssim 5$, the phenomenology of the heavier $A, H, H^{ \pm}$bosons is richer [9]. For the cross sections, we display in Fig. 8 (left) the rates for the relevant processes at the LHC with $\sqrt{s}=8 \mathrm{TeV}$ assuming $\tan \beta=2.5$. For smaller $\tan \beta$ values, the rates except for $p p \rightarrow H / A+b \bar{b}$ are even larger as the $H / A+t t$ and $H V V$ couplings are less suppressed. The $g g \rightarrow H / A$ processes give significant rates at $M_{\Phi} \lesssim 500 \mathrm{GeV}$ and the other modes have much smaller rates.

The $H / A$ decay pattern, is involved at low $\tan \beta$ as can be seen in Fig. 8 where the branching ratios are shown for $\tan \beta=2.5$. Above the $t \bar{t}$ threshold, the channels $H / A \rightarrow t \bar{t}$ are by far dominant for $\tan \beta \lesssim 3$ and do not leave space for any other mode. Below the $t \bar{t}$ threshold, the $H \rightarrow W W, Z Z$ mode is still significant while for $2 M_{h}\left(M_{h}+M_{Z}\right) \lesssim M_{H} \lesssim 2 m_{t}$ the decays $H \rightarrow h h(A \rightarrow Z h)$ are important. Hence, many decay and production channels need to be considered in this low $\tan \beta$ regime.
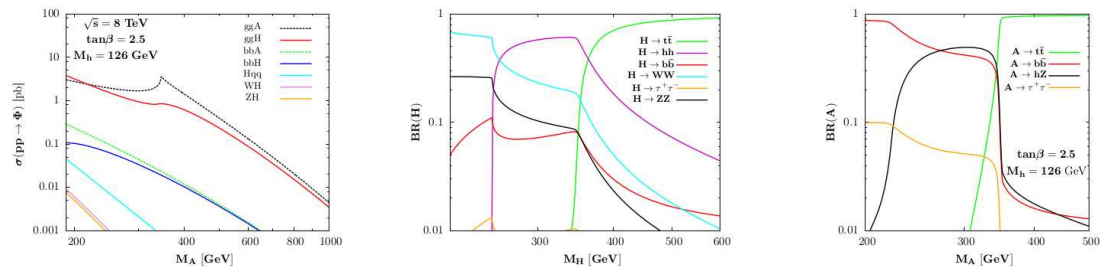

Figure 8. The MSSM heavier neutral Higgs boson cross sections at the LHC at $\sqrt{s}=8$ (left) and their decay decay branching ratios (center and right) for $\tan \beta=2.5$. They are obtained with the programs of Ref. [21].

\subsection{Constraints from the LHC Higgs searches}

Searches for the by far efficient channel $p p \rightarrow g g+b b \rightarrow H / A \rightarrow \tau^{+} \tau^{-}$have been performed by ATLAS and CMS with at the $7+8 \mathrm{TeV}$ runs. Upper limits on the production cross section times decay branching ratio have been set and they can be turned into constraints on the MSSM parameter space. In Fig. 9 (left), displayed is the sensitivity of the CMS analysis of Ref. [22] in the [ $\tan \beta, M_{A}$ ] plane. At $95 \% \mathrm{CL}$, the mass range $M_{A} \lesssim 300 \mathrm{GeV}$ for $\tan \beta \gtrsim 7-10$, extending up to the range $M_{A} \lesssim 700 \mathrm{GeV}$ for $\tan \beta \gtrsim 20$. In the right panel of Fig. 9, the limits from the latest ATLAS analysis with the $8 \mathrm{TeV}$ full data of the charged Higgs search in the mode $t \rightarrow b H^{+} \rightarrow b \tau^{+} v$ [23] is shown. For all values of $\tan \beta$, these searches now practically rule out the mass range $M_{H^{ \pm}} \lesssim 160 \mathrm{GeV}$, which approximately corresponds to $M_{A} \lesssim 140 \mathrm{GeV}$. Similar results are found by the competing experiments.

The reopening of the low $\tan \beta$ region allows to consider a plethora of very interesting channels for the heavier Higgs bosons to be also investigated at the LHC: heavier CP-even $H$ decays into massive gauge bosons $H \rightarrow W W, Z Z$ and Higgs bosons $H \rightarrow h h, \mathrm{CP}-$ odd Higgs decays into a vector and a Higgs boson, $A \rightarrow h Z, \mathrm{CP}-$ even and CP-odd Higgs decays into top quarks, $H / A \rightarrow t \bar{t}$, and even the charged Higgs decay $H^{ \pm} \rightarrow W h$. These final states have been searched for by ATLAS and CMS in the context of a heavy SM Higgs boson or for new resonances in some non-SUSY beyond the SM scenarios and the analyses can be adapted to the case of the heavier MSSM Higgs bosons. They would then allow to cover a larger part of the parameter space of the MSSM Higgs sector in a model-independent way, i.e. without using the information on the scale $M_{S}$ and more generally on the SUSY particle spectrum that appear in the radiative corrections. 

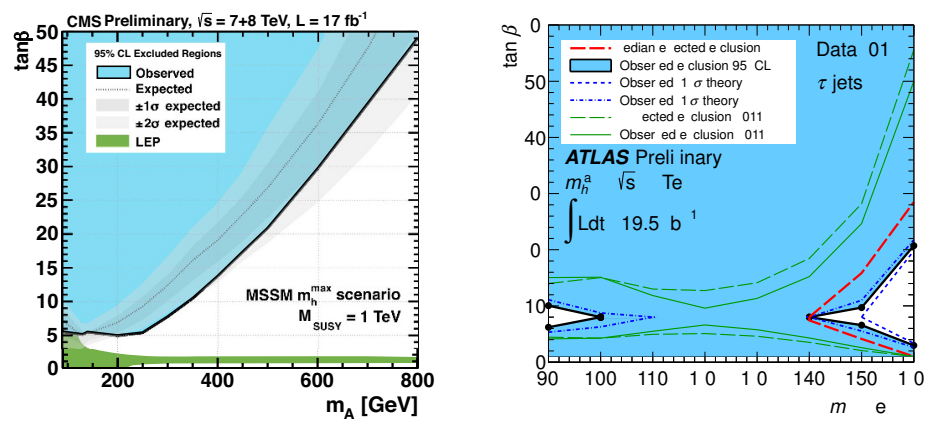

Figure 9. The exclusion limits in the MSSM plane from the channel $p p \rightarrow h / H / A \rightarrow \tau^{+} \tau^{-}$by CMS (left) [22] and from the channel $t \rightarrow b H^{+} \rightarrow b \tau v$ by ATLAS (right) [23].

In Ref. [9] a ṕreliminary analysis of these channels has been performed using current information given by the ATLAS and CMS collaborations in the context of searches for the SM Higgs boson or other heavy resonances (in particular new $Z^{\prime}$ or Kaluza-Klein gauge bosons that decay into $t \bar{t}$ pairs). The results are shown in Fig. 10 with an extrapolation to the full $25 \mathrm{fb}^{-1}$ data of the $7+8 \mathrm{TeV}$ LHC run (it has been assumed that the sensitivity scales simply as the square root of the number of events). The sensitivities from the usual $H / A \rightarrow \tau^{+} \tau^{-}$and $t \rightarrow b H^{+} \rightarrow b \tau v$ channels are also shown. The green and red areas correspond to the domains where the $H \rightarrow V V$ and $H / A \rightarrow t \bar{t}$ channels become constraining. The sensitivities in the $H \rightarrow h h$ and $A \rightarrow h Z$ modes are given by, respectively, the yellow and brown areas which peak in the mass range $M_{A}=250-350 \mathrm{GeV}$ that is visible at low $\tan \beta$.

The outcome is impressive. These channels, in particular $H \rightarrow V V$ and $H / A \rightarrow t \bar{t}$, are very constraining as they cover the entire low $\tan \beta$ area up to $M_{A} \approx 500 \mathrm{GeV}$. Even $A \rightarrow h Z$ and $H \rightarrow h h$ would be visible at the current LHC in small portions of the parameter space.

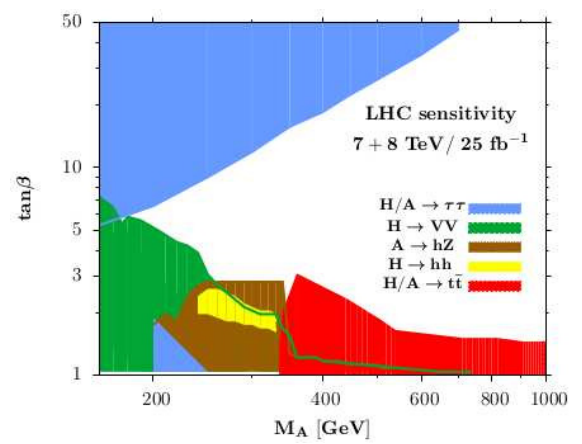

Figure 10. The estimated sensitivities at $\mathrm{LHC}$ with $7+8 \mathrm{TeV}$ and $25 \mathrm{fb}^{-1}$ data in the various search channels for the heavier Higgs bosons in the [ $\tan \beta, M_{A}$ ] plane: $H / A \rightarrow \tau \tau, H \rightarrow W W+Z Z, H / A \rightarrow t \bar{t}, A \rightarrow h Z$ and $H \rightarrow h h$ [9].

\section{Conclusions}

The discovery of the Higgs boson and the non-observation of new particles at the LHC has far reaching consequences for supersymmetric theories and, in particular, for their simplest low energy formulation, the MSSM. The mass of approximately $125 \mathrm{GeV}$ of the observed state implies that the scale of SUSY-breaking is rather high, at least $O(\mathrm{TeV})$. This is backed up by the limits on the masses of strongly interacting SUSY particles set by the ATLAS and CMS searches, which in most cases exceed the TeV range. This implies that if SUSY is indeed behind the stabilization the Higgs mass against very high scales that enter via quantum corrections, it is either fine-tuned at the permille level at least or its low energy manifestation is more complicated than expected. 
The LHC Higgs data also indicate that the couplings of the observed state to fermions and gauge bosons are approximately SM-like. In the context of the MSSM, this implies that we seem to be in the decoupling regime and this $125 \mathrm{GeV}$ particle can be only identified with the lightest $h$ boson, while the other $H / A / H^{ \pm}$states must be heavier than approximately the Fermi scale. This last feature is also backed up by the constraints from direct searches of these heavier Higgs states at the LHC.

We do hope that the upcoming energy upgrade of the LHC to $\approx 14 \mathrm{TeV}$ and the planed order of magnitude (or more) increase in luminosity will allow to perform more precise measurements of the Higgs properties and to conduct more powerful searches for heavier states. These would allow to probe much higher mass scales than presently, allowing hopefully for the first hints of the MSSM to be detected, at last.

Acknowledgements:I would like to thank the organisers of this conference in Kolymbari, in particular our three "muses", for their invitation and for setting such a nice atmosphere during the meeting.

\section{References}

[1] ATLAS collab., Phys. Lett. B716 (2012) 1; CMS collab., Phys. Lett. B716 (2012) 30.

[2] K. Olive et al., Particle Data Group, Chin. Phys. C38 (2014) 090001.

[3] See the talk of S. Gascon-Shotkin, these proceedings.

[4] F. Englert and R. Brout, Phys. Rev. Lett. 13 (1964) 321; P. Higgs, Phys. Rev. Lett. 13 (1964) 508; G. Guralnik, C. Hagen and T. Kibble, Phys. Rev. Lett. 13 (1964) 585.

[5] For reviews of Higgs physics in the context of the SM and the MSSM, see for instance: A. Djouadi, Phys. Rept. 457 (2008) 1; Phys. Rept. 459 (2008) 1; Eur. Phys. J. C74 (2014) 2704.

[6] M. Drees, R. Godbole and P. Roy, Theory and phenomenology of sparticles, World Sci., 2005.

[7] M. Carena, H. Haber, Prog. Part. Nucl. Phys. 50 (2003) 63; B. Allanach et al., JHEP 0409 (2004)

044; S. Heinemeyer, W. Hollik and G. Weiglein, Phys. Rept. 425 (2006) 265.

[8] M. Carena et al., Eur. Phys. J. C73 (2013) 2552.

[9] A. Djouadi and J. Quevillon, JHEP 1310 (2013) 028.

[10] L. Maiani, A. Polosa and V. Riquer, Phys. Lett. B718 (2012) 465; Phys. Lett. B724 (2013) 274.

[11] A. Djouadi, L. Maiani et al., Eur. Phys. J. C73 (2013) 2650.

[12] MSSM Working Group, hep-ph/9901246; A. Djouadi, J.L. Kneur and G. Moultaka, Comput. Phys. Commun. 176 (2007) 426; M. Muhlleitner et al., Comput. Phys. Commun. 168 (2005) 46.

[13] A. Arbey et al., Phys. Lett. B708 (2012) 162.

[14] N. Bernal, A. Djouadi and P. Slavich, JHEP 0707 (2007) 016.

[15] A. Arbey et al., JHEP 1209 (2012) 107; Phys. Lett. B720 (2013) 153.

[16] See e.g., M. Carena et al., Nucl. Phys. B577 (2000) 88.

[17] A. Djouadi, Phys. Lett. B435 (1998) 101; A. Djouadi, V. Driesen, W. Hollik et al., Eur. Phys. J. C1 (1998) 149; Eur. Phys. J. C1 (1998) 163.

[18] A. Djouadi et al., Phys. Lett. B709 (2012) 65; Eur. Phys. J. C73 (2013) 2455.

[19] S. Dittmaier et al., (LHC Higgs cross section working group), arXiv:1101.0593.

[20] J. Baglio et al., JHEP 1010 (2010) 064; JHEP 1103 (2011) 055; Phys. Lett. B716 (2012) 203.

[21] M. Spira et al., Comput. Phys. Commun. 108 (1998) 56; Acta. Phys. Polon. B38 (2007) 635;

Nucl. Phys. B453 (1995) 17; Fortschr. Phys. 46 (1998) 203; hep-ph/9510347.

[22] The CMS collaboration, CMS-PAS-HIG-12-050.

[23] The ATLAS collaboration, ATLAS-CONF-2013-090. 